\title{
sciendo
}

\section{The Relationship Between Pistol Olympic Shooting Performance, Handgrip and Shoulder Abduction Strength}

\author{
by \\ Daniel Mon-López ${ }^{1}$, Maria S. Zakynthinaki², Carlos Alberto Cordente', \\ Jorge García-González ${ }^{3}$
}

\begin{abstract}
The ability to stabilize the gun, a crucial factor for performance in air pistol Olympic shooting, is thought to be strongly related to the muscular work of the shooter's shoulder and forearm. The objective of the present study was to confirm this relationship by analysing the influence of maximal finger flexor forces and maximal isometric shoulder force on performance in female air pistol shooting. Twenty-three female pistol shooters participated in the study. Handgrip and shoulder force data were recorded under competition conditions, during the official training time of national Spanish championships on the day previous to the competition and at the official training stands. Performance was measured as the total score of 40 shots at competition. Linear regressions between performance and age, weight, height, training experience, body mass index (BMI), mean and maximal finger flexor and shoulder forces were calculated. Significant correlations were found between performance and a) training hours $b$ ) peak finger flexor force relative to the BMI, and c) peak isometric force of the shoulder abduction relative to the BMI. The study concludes that there is a statistically significant correlation between performance and muscular forces exerted by the athletes relative to their BMI. Appropriate muscular strength training programs are therefore necessary in female air pistol Olympic shooting.
\end{abstract}

Key words: score, deltoid force, dynamometer, BMI, performance.

\section{Introduction}

There are many factors that affect performance in Olympic shooting. Elite shooters are capable of reaching extreme levels of precision and accuracy. In female air pistol shooting, the best shooters often reach scores as high as the current world record (393/400), or in other words success of $98.2 \%$ hitting the ten points score. Considering that the maximum score of a shot (10 points) represents accuracy within a circle of a diameter of $11.5 \mathrm{~mm} \pm 0.1 \mathrm{~mm}$ from a shooting distance of 10 meters, failure or important loss in performance due to uncontrolled movements becomes obvious (International Shooting Sport Federation [ISSF], 2014).

It is widely agreed that the ability to stabilize the gun is crucial for performance in pistol shooting (Pellegrini and Schena, 2005) and one of the most important factors that predict performance when the shot is within the circle that corresponds to a score of 9 points (Hawkins, 2011). This ability to stabilize the gun is affected by two factors: the movements of the center of pressure (COP) that reach the gun through the kinetic chains of the body (Pellegrini and Schena, 2005) and the movements caused by muscular tremor (Lakie, 2010) that are related to various

\footnotetext{
1 - Sports Department, Faculty of Physical Activity and Sport Sciences (INEF), Universidad Politécnica de Madrid, Madrid, Spain.

2 - Department of Electronics, University of Applied Sciences (TEI) of Crete, 73134 Chania, Greece.

3 - Social Sciences Department, Faculty of Physical Activity and Sport Sciences (INEF), Universidad Politécnica de Madrid, Madrid, Spain.
} 
factors and also to different types of muscular contractions. Furthermore, this tremor depends on both the preparatory heart rate patterns and the heart rate variability (HRV) before shooting which in turn can be influenced by the shooter's physiological state, the levels of anxiety, as well as the changes in anxiety levels (Kayihan et al., 2014; Konttinen et al., 1989; Ortega and Wang, 2018).

According to Pellegrini and Schena (2005) the ability to stabilize the gun is determined by muscular work of the shoulder and the forearm. The study of Tang et al. (2008) also reports that elite shooters are able to better stabilize the gun and the wrist by minimizing muscular tremor, possibly via the appropriate co-activation of the hand muscles.

The studies that refer to the important role of the physical and muscular condition of an Olympic shooter are limited. Vercruyssen et al. (1989) report that performance in pistol shooting is closely related to wrist and deltoid strength $(r=$ .82-.89) that also depend on the time the athlete has to shoot, which varies between shooting disciplines. Anderson and Plecas (2000) also found that the maximal contracting force of the palmar flexor muscles was related to performance $(r=.38)$, in heterogeneous groups of male and female pistol shooters. However, more recent studies show small percentages of influence between handgrip strength $(r=.24)$ (Kayihan et al., 2013), finger flexor and shoulder abduction isometric forces and performance (adjusted $r^{2}=$ .04-.11) associated at the same time with variant coefficients of $2 \%$ of the performance on males (Mon et al., 2015).

Specialized books and journals often refer to the necessity and the importance of physical conditioning (Mon et al., 2016; Mon et al., 2014) as well as workouts that strengthen the muscles that take part in pistol shooting (Reinkemeier and Bühlmann, 2013). The study of Krasilshchikov et al. (2007) supports these recommendations: a physical conditioning program combined with technique training was found to increase the ability to stabilize the gun and therefore to improve performance. Other authors such as Mason et al. (1990) however, found no relationship between grip strength and performance in pistol shooting. No consensus exists therefore in the scientific literature regarding the influence of the physical condition of pistol shooters on their performance.

It should be finally noted that most of the existing studies are based on data recorded under laboratory or training conditions. Very few studies can be found in the literature referring to performance under actual competition conditions (Ihalainen et al., 2017; Mon et al., 2015).

Therefore, the objective of the present study was to examine and confirm the influence of maximal finger flexor forces and maximal isometric shoulder force on performance in female air pistol shooting, via the analysis of data recorded under competition conditions.

\section{Methods}

\section{Participants}

Twenty-three female pistol shooters participated in this study. The mean \pm standard deviation values of the group characteristics are shown in Table 1. All participants took part in the national Spanish championships of air pistol shooting.

Participation in the study was voluntary and open to all competing shooters during the day previous to competition. According to the regulations of the Spanish Federation of Olympic Shooting, eligibility to compete required a previously obtained (in other national competitions) minimum score of 320 points in women air pistol.

The ethics committee of the Technical University of Madrid provided an approval for the present study and an informed consent form was signed by all participants before data collection. We confirm that our research meets the highest ethical standards for authors and coauthors. The study was performed following the guidelines of the Declaration of Helsinki, last modified in 2008.

\section{Measures}

The descriptive variables regarding the participants' profile and strength were measured as follows:

Age (years), experience (years) and training (hours/week) were taken with a questionnaire filled by the participants.

Body weight was measured in underclothes to the nearest $100 \mathrm{~g}$ with a force plate (Kistler Force Plate 9286AA; Kistler Holding AG, Winterthur, Switzerland).

Body height was measured twice to the 
nearest $5 \mathrm{~mm}$ using a portable stadiometer (Seca 206; Seca gmbh \& co., Hamburg, Germany) with the mean values used for further analysis.

The body mass index (BMI) was calculated using the following formula: weight $(\mathrm{kg})$ divided by height $(\mathrm{m})$ squared.

Shooting performance was evaluated during 40 shots under competition conditions and measured using official paper targets, according to the Spanish Shooting Federation Rules and Regulations and as provided by the referees of the Spanish Olympic Shooting Federation after the competition.

Peak finger flexor force was the personal best of the three attempts, while mean finger flexor force was the average of the three attempts.

Peak and mean finger flexor force values relative to the BMI were also calculated.

Peak isometric force of the shoulder abduction was the personal best of the three attempts, while mean isometric force of the shoulder abduction was the average of the three attempts

Peak and mean isometric force values of the shoulder abduction relative to the BMI were also calculated.

\section{Design and Procedures}

The protocol consisted of two tests performed three times each. Both tests were carried out on the day previous to the competition and during the official training time. During the first test a maximal isometric contraction of shoulder abduction was performed, starting from an initial position of $90^{\circ}$ shoulder abduction (similar to the real pistol shooting position). To calculate the force exerted by the shooter, the minimum weight measured by the plate during the test was subtracted from the $16 \mathrm{~kg}$ of the weight placed on it.

During the second test, the arm normally used by the shooter was extended along the body and the hand dynamometer was used to measure the finger flexor forces. The participants were asked to perform a maximal contraction of the finger flexors without bending the elbow. A hand dynamometer (Takei Hand Grip Dynamometer A5401; Takei Scientific Instruments, Niigata, Japan) was used to measure the maximal finger flexor force. In order to measure the maximal isometric strength of the shoulder, a $16 \mathrm{~kg}$ weight was attached to a force plate (Kistler Force Plate
9286AA) by means of a rigid steel cable the length of which was adaptable to the height of the participants.

Taking into account characteristics of such a maximal force test, a minimum rest of $60 \mathrm{~s}$ was allowed between measurements for both tests (de Salles et al., 2009).

Statistical Analysis

The Kolmogorov-Smirnov test was used to determine the goodness of fit to the normal distribution of the variables. To examine the relationships between performance and maximal muscular forces, simple linear regression was used. The level of significance was set at $p<.05$. The statistical analysis of the variables was performed using SPSS Statistics 17.

\section{Results}

Table 2 presents the mean ( \pm standard deviation) of the shooters' data regarding performance, average and maximum forces.

Significant correlations were found between performance and training hours $F(1,21)=$ $5.43, p=.030$, BMI $F(1,21)=5.74, p=.026$, mean finger flexor force relative to the BMI $F(1,21)=$ $4.55, p=.045$, peak finger flexor force relative to the BMI $F(1,21)=4.39, p=.048$, mean isometric force of shoulder abduction relative to the BMI $F(1,21)=6.89, p=.016$, peak isometric force of shoulder abduction relative to the BMI $F(1,21)=$ $4.90, p=.038$, and mean isometric force of shoulder abduction relative to the weight $F(1,21)=$ $6.16, p=.022$. No significant correlations were found between performance and the rest of the strength variables $(p>$.05). No statistically significant correlations were found between performance and age, weight or height $(p>.05)$.

\section{Discussion}

The ability to stabilize the gun is crucial for achieving high scores in Olympic pistol shooting, as some studies confirm this phenomenon (Gulbinskienė and Skarbalius, 2009; Hawkins, 2011). Movements of the pistol, generally induced by body movements (Pellegrini and Schena, 2005), have been reported to be significantly correlated with muscular factors of the wrist and the shoulder (Pellegrini and Schena, 2005; Tang et al., 2008). 


\section{Table 1}

Mean and standard deviation of the variables that refer to the participants' profile

\begin{tabular}{lccc}
\hline & Mean & & SD \\
\hline Age (years) & 31.26 & \pm & 11.21 \\
Body height (m) & 1.64 & \pm & 0.06 \\
Body mass (kg) & 66.58 & \pm & 12.22 \\
& & & \\
BMI (kg/m $\left.{ }^{2}\right)$ & 24.63 & \pm & 4.28 \\
Experience (years) & 9.09 & \pm & 6.43 \\
Training (hours per week) & 7.13 & \pm & 9.15 \\
Performance (points) & 359.39 & \pm & 14.29 \\
\hline
\end{tabular}

Table 2

Mean and standard deviation of the variables that refer to the participants' strength

\begin{tabular}{|c|c|c|c|}
\hline & Mean & & SD \\
\hline Mean finger flexor force $(\mathrm{N})$ & 272.82 & \pm & 53.86 \\
\hline Peak finger flexor force $(\mathrm{N})$ & 288.02 & \pm & 51.01 \\
\hline Mean finger flexor force relative to the BMI & 116.96 & \pm & 31.21 \\
\hline Peak finger flexor force relative to the BMI & 122.72 & \pm & 29.74 \\
\hline Mean shoulder isometric force $(\mathrm{N})$ & 52.09 & \pm & 13.54 \\
\hline Peak shoulder isometric force $(\mathrm{N})$ & 53.96 & \pm & 14.13 \\
\hline Mean shoulder isometric force relative to the BMI & 22.06 & \pm & 6.40 \\
\hline Peak shoulder isometric force relative to the BMI & 22.78 & \pm & 6.51 \\
\hline
\end{tabular}


Table 3

Linear regression between performance and training, experience, BMI (Body Mass Index), mean and peak finger flexor force, mean and peak finger flexor force relative to the BMI, mean and peak shoulder isometric abduction force, mean and peak shoulder isometric abduction force relative to the BMI and mean shoulder isometric abduction force relative to the weight.

\begin{tabular}{|c|c|c|c|c|}
\hline & $r$ & Adjusted $r^{2}$ & $\begin{array}{c}\mathrm{CV} \%=\mathrm{SD} / \mathrm{M} \\
\times 100\end{array}$ & sig \\
\hline Training & .46 & .17 & 3.67 & $.030^{*}$ \\
\hline Experience & .15 & .02 & 3.00 & .483 \\
\hline BMI & .46 & .18 & 3.60 & $.026^{*}$ \\
\hline Mean finger flexor force & .23 & .01 & 3.96 & .299 \\
\hline Peak finger flexor force & .19 & .01 & 3.99 & .386 \\
\hline Mean finger flexor force relative to the BMI & .42 & .14 & 3.69 & $.045^{*}$ \\
\hline Peak finger flexor force relative to the BMI & .42 & .13 & 3.70 & $.048^{*}$ \\
\hline Mean shoulder isometric abduction force & .30 & .04 & 3.88 & .170 \\
\hline Peak shoulder isometric abduction force & .20 & .00 & 3.98 & .353 \\
\hline $\begin{array}{l}\text { Mean shoulder isometric abduction force } \\
\text { relative to the BMI }\end{array}$ & .50 & .21 & 3.53 & $.016^{*}$ \\
\hline $\begin{array}{l}\text { Peak shoulder isometric abduction force relative } \\
\text { to the BMI }\end{array}$ & .44 & .15 & 3.66 & $.038^{*}$ \\
\hline $\begin{array}{l}\text { Mean shoulder isometric abduction force } \\
\text { relative to the weight }\end{array}$ & .48 & .19 & 3.59 & $.022^{*}$ \\
\hline
\end{tabular}

Level of significance $p<.05$.

Specifically in pistol shooting, it has been shown that elite shooters' coordinative control of the arm is superior to their lower level competitors, with "smaller tremor amplitudes" in the hand and the pistol.

This ability to minimize muscular tremor could be due to the correct and coordinated coactivation of the muscles involved in the shot
(Tang et al., 2008). In addition, this final coordination control is closely related to other factors such as movements of the COP that affect stability of the gun (Pellegrini and Schena, 2005) which can be in turn influenced by other hormonal factors, muscle temperature, previous exercise or conditions of localized ischemia (Lakie, 2010). Anxiety also plays a very important 
role in the ability to stabilize the gun and therefore performance, as it modifies the cardiorespiratory rhythms and increases muscular tremor (Kayihan et al., 2014).

Regarding athletes' physical strength, although it is not easy to determine the relationship of a shooter's overall physical condition with shooting performance, the analysis presented here suggests that, in female air pistol shooting, there is a close relationship between performance and muscular forces exerted by athletes relative to their BMI. Similar results, but with less influence, were found in the literature relating performance and the BMI to Olympic pistol modalities in men. However, the influence of the force relative to weight was more consistent in men than in women (Mon et al., 2015). Furthermore, we did not find any correlation between performance and absolute force values, neither shoulder or finger flexor force. These results are contrary to those of Mon et al. (2015) who found a relationship between absolute finger flexor force and performance in men. Taking these into account we could conclude that the way muscular strength affects performance differs between male and female pistol shooters. Coaches should consider this differentiation when designing appropriate muscular strength training programs.

We found that the mean value of the score achieved during competition can be explained as follows: $15-21 \%$ of the variance of performance is explained by the force exerted by the shoulders and $13-14 \%$ by the force exerted by the hand. These results are in agreement with the results of Anderson and Plecas (2000), who found a $r^{2}=.14$ correlation between performance and finger flexor force (similar results). Our results are also in agreement with the results of Vercruyssen et al. (1989), who found a correlation of $r^{2}=.67-.79$ between performance and muscular forces exerted by the fingers as well as the shoulder. This correlation is much higher than the one found in the present study, the final conclusion is, however, similar: force exerted by the shoulder is more relevant for performance than force exerted by the hand of the shooter. It should be mentioned here that Mason et al. (1990) found no relationship between finger flexor forces and performance probably because of the protocol used to measure the forces or variables selected for analysis which were different from the ones used in our study.

In other sports the relationship between performance and a strength weight ratio has been documented (Wall et al., 2004). The results of the present study show that in female air pistol shooting there is also a relationship between performance and the mean isometric shoulder abduction force relative to body mass.

Our results are also in agreement with the practical suggestions of various professionals, found in specialized journals and books, regarding the importance of physical conditioning (Mon et al., 2016; Mon et al., 2014) and resistance training (Reinkemeier and Bühlmann, 2013) for improving performance in Olympic shooting. We also showed that the number of weekly training hours is also important for performace, in acordance with the studies of Goonetilleke et al. (2009) and Krasilshchikov et al. (2007).

Finally, it should be noted that the present study is based on data recorded under competition conditions (during the official training period just before the championships) and not in a laboratory setting or during training, as is the case with other studies, thus associating our data with the participants' actual performance in competition. This fact, however, affected the study's statistical power as the number of participants was limited: some of the competitors denied participation as they thought that the test would influence their performance (something that should be analyzed in future studies).

According to analysis of the world championships from 1998 to now, the $\mathrm{CV}=3-3.7$ found in the present study corresponds to a difference of 12-15 points of the total competition score (maximum 400 points), which is equivalent to an average difference of $65-80$ positions in the final ranking. Considering also the need for precision in Olympic pistol shooting that reaches accuracy levels as high as $98.2 \%$ (ISSF, 2014), we believe that the correlations obtained with the present analysis, in relation with the forces exerted by the shooters, should be taken into account for improvements in performance.

As a conclusion, we showed a direct correlation between shooting performance under competition conditions and relative handgrip strength as well as relative isometric shoulder abduction strength. We may conclude that 
training programs to improve relative handgrip strength and relative isometric shoulder abduction strength are necessary for optimizing performance in female air pistol Olympic shooting.

\section{Acknowledgments}

The authors would like to acknowledge the Autonomous University of Madrid and the Technical University of Madrid for their financial support.

\section{References}

Anderson GS, Plecas DB. Predicting shooting scores from physical performance data. Policing, 2000; 23: 525537

de Salles BF, Miranda F, da Silva Novaes J, Lemos A, Willardson JM. Rest interval between sets in strength training. Sports Med, 2009; 39: 765-777

Goonetilleke RS, Hoffmann ER, Lau WC. Pistol shooting accuracy as dependent on experience, eyes being opened and available viewing time. Appl Ergon, 2009; 40: 500-508

Gulbinskienè V, Skarbalius A. Peculiarities of investigated characteristics of lithuanian pistol and rifle shooters' training and sport performance. Ugdymas Kuno Kultura, 2009; 21

Hawkins R. Identifying mechanic measures that best predict air-pistol shooting performance. Int J Perf Anal Spor, 2011; 11: 499-509

Ihalainen S, Mononen K, Linnamo V, Kuitunen S. Which technical factors explain competition performance in air rifle shooting? Int J Sports Sci Coa, 2017; 78-85

International Shooting Sport Federation. World records, 2014. Available at: http://www.issfsports.org/results/records/world_records.ashx; accessed on 02.02.2014

Kayihan G, Ersoz G, Özkan A, Mitat K. Relationship between efficiency of pistol shooting and selected physical-physiological parameters of police. Policing, 2013; 36: 819-832

Kayihan G, Ersoz G, Özkan A, Tuna M. Relationship between anxiety, heart rate and efficiency of pistol shooting. J Hum Sci, 2014; 11: 1266-81

Konttinen N, Lyytinen H, Viitasalo J. Preparatory heart rate patterns in competitive rifle shooting. J Sport Sci, 1998; 16: 235-42

Krasilshchikov O, Zuraidee E, Singh R. Effect of general and auxiliary conditioning on specific fitness of young pistol and rifle shooters. Asian J. Exerc. Sport Sci, 2007; 4: 01-06

Lakie M. The influence of muscle tremor on shooting performance. Exp Physiol, 2010; 95: 441-450

Mason B, Cowan L, Gonczol T. Factors affecting accuracy in pistol shooting. Excel, 1990; 6: 2-6

Mon D, Zakynthinaki MS, Cordente CA, Antón AJM, Rodríguez BR, Jiménez DL. Finger Flexor Force Influences Performance in Senior Male Air Pistol Olympic Shooting. PLoS ONE, 2015; 10: e0129862

Mon D, Zakynthinaki MS, Cordente CA, Barriopedro MI, Sampedro J. Prevalidation of body sway shooting test without the use of weapons. Rev Int Med Cienc Ac, 2016; 64: 775-787

Mon D, Zakynthinaki MS, Cordente CA, Monroy A, López D. Validation of a Dumbbell Body Sway Test in Olympic Air Pistol Shooting. PLoS ONE, 2014; 4: e96106

Ortega E, Wang CJK. Pre-performance Physiological State: Heart Rate Variability as a Predictor of Shooting Performance. Appl Psychophysiol Biofeedback, 2018; 43: 75-85 
Pellegrini B, Schena F. Characterization of arm-gun movement during air pistol aiming phase. J Sport Med Phys Fit, 2005; 45: 467-475

Reinkemeier H., Bühlmann G. Pistol Shooting - The Olympic Disciplines. Dortmund : MEC High Tech Shooting Equipment; 2013

Tang WT, Zhang WY, Huang CC, Young MS, Hwang IS. Postural tremor and control of the upper limb in air pistol shooters. J. Sports Sci., 2008; 26: 1579-1587

Vercruyssen M, Christina RW, Muller E. Relationship of strength and precision in shooting activities. J Hum Ergol (Tokyo), 1989; 18: 153-168

Wall CB, Starek JE, Fleck SJ, Byrnes WC. Prediction of indoor climbing performance in women rock climbers. J Strength Cond Res, 2004; 18: 77-83

\section{Corresponding author:}

\section{Daniel Mon}

Sports Department, Faculty of Physical Activity and Sport Sciences (INEF), Universidad Politécnica de Madrid, Madrid, Spain

c/ Martín Fierro 7, 28040. Madrid. Spain.

Telephone: +34910677869

E-mail: daniel.mon@upm.es 